\title{
The secret recipe to structure a good research
}

\section{paper}

\section{Editorial}

It is my pleasure to write this editorial for journal of Biosensors \& Bioelectronics. The secret recipe to structure a good research paper lies in the ingredients of the paper. A good research paper conveys an idea presented effectively with its validation and discussions. The main ingredients of a good research paper are "Title, Abstract, Keywords, followed by Introduction, Methods, Results, and Discussion (IMRAD), Conclusion, Future scope of work, Acknowledgement, References and Appendix or supplementary data". However, depending upon the nature and theme of the research paper the author may add or remove few more ingredients like literature review, related work, experimental setup details etc. to spice up its research paper. Further while structuring a good research papers there are some key points to be kept in mind. Firstly, while writing a research paper always try to follow the aims and scope of the journal, because a research paper which does not stick to the aim and scope of the journal is likely to get rejected from the editor's desk itself before going to review. Therefore, before selecting journal for prospective publication of a research paper always ensure that the paper comes under the aim and scope of the journal, check the types of articles already published or accepted for publication in the journal, readership of the journal. While carrying out the literature survey consider all the previous publications done in the field of your research paper. Make an exhaustive study of the related literature available. Give proper citations and credits throughout the paper and adhere to publication ethics. While quoting work from others paper do not copy sentences verbatim and avoid plagiarism of others' work. Always try to give proper Citations and acknowledge others' work appropriately. While formatting and editing the paper adhere to the Guidelines for the Authors given by the journal. Generally, editors do not like wasting time on poorly prepared manuscripts and poorly prepared scripts has more chances to get rejected right from the desk of the editor. Another important aspect of a research paper are its results. Figures, graphs and tables are the most efficient way to present results. Try to use descriptive captions and legends to make figures and tables self-explanatory. Provide thorough discussions of all the experimental results, graphs, tables, figures etc. Many times, there is repetition or duplication of results done in graphs and tables or illustrations and text. Avoid any sort of duplication of results which has already been described in text or any other illustrations. Wherever possible Compare results with existing contemporary techniques. While writing paper ensure appropriate usage of the language. The editors/reviewers of journals do not correct language or grammar of the paper they will only point it out, it is the author's responsibility to correct it. Poorly written and inappropriate usage of language makes it difficult for the editors and reviewers to properly comprehend your paper and there are more chances that your paper may get rejected. If English is not your first language you can take help of someone who is proficient in editing. Avoid common mistakes while constructing sentences, maintain coherence between sentences and paragraphs, avoid too long sentences, use correct tenses, ensure proper grammar, avoid mixing of languages. English language should be used throughout the manuscript, including figures, charts, graphs and photos. When you are ready to submit go through the draft of your paper, revise it thoroughly before final submission. you can

\author{
Volume 2 Issue 2 - 2018
}

\author{
Kshitij Shinghal \\ Department of Electronics \& Communication engineering, India
}

Correspondence: Department of Electronics \&

Communication engineering, Moradabad Institute of Technology, India,Email: kshinghal@gmail.com

Received: March 18, 2018 | Published: March 22, 2018

also take help of your colleagues for English editing and checking your final draft. Generally about $40 \%$ of all submitted manuscripts are rejected before peer review. Therefore, always make it a point to revise manuscript before final submission.

MedCrave Online Journal of Applied Bionics and Biomechanics (MOJABB) is an International, Peer reviewed Journal of advanced technological developments based on the science of biological systems. The journal solicits research papers for its forthcoming issue in all aspects of bionic science and engineering including fundamental understandings of animals and plants for bionic engineering, such as locomotion and behaviors of animals, structures, composites, morphology and physical properties of plants and natural materials, applications of such understandings in engineering, technology and designs. Journal accepts Research Papers, Review Articles, Short Communications, Case Reports, Mini-Reviews, Opinions, Letter to Editors, etc. in this field which will be enlightening the scientific community. On behalf of the editorial team of the journal I invite authors from all over the world to share their innovative research and findings. Further I assure that the contents submitted to MedCrave will enjoy more visibility and will be peer reviewed too. You can submit your works on any topic of your expertise within the scope of journal. Journal ensures to share only high-quality content, so there is no room for copied or doubtful content to be published. You can submit work on any topic relevant to science. The content should be unique, original and the presentation must be of potential interest to the readers. You can submit your research articles too. These research papers must be original and must be in the major field of science. The research articles can include the findings and the methodology you used. You can also compile your evidences that lead to your conclusions. So once again I beseech timely submission of research papers for prospective publication in upcoming issue of the journal.

\section{References and Acknowledgements}

I have based this paper on the materials collected from several courses I've attended. Some of this information is also featured in various tutorials available online. In addition, I have also consulted several web pages while writing this article. I would also like to thank Mr. Amit Saxena and Ms. Deepti Shinghal for their valuable support, without their help this article would have been impossible to complete. 